\title{
La Demanda de Autonomía Tecnológica en la Educación a Distancia: una Aproximación en el Contexto de la UNED
}

\author{
(The Demand of Technological Autonomy in Distance Education: an Approach in the \\ Context of the UNED)
}

\author{
JAVIER CALLEJO \\ (UNED, España)
}

\begin{abstract}
RESUMEN: Este artículo analiza la relevancia de la demanda de autonomía e independencia en la enseñanza como factor que atrae alumnos a las universidades a distancia. Se observa que es una demanda que ha crecido en los últimos años y que es previsible que siga creciendo en el futuro. Aun cuando su extensión es general, la demanda de autonomía se encuentra especialmente presente en algunos sectores de alumnos. Posteriormente se ha concretado la demanda de autonomía en lo que se ha denominado autonomía tecnológica. Es decir, una autonomía que prescinde totalmente de la relación presencial con el profesor. Por lo tanto, la relación se concentra en el uso de los diversos medios de comunicación y los exámenes. Según nuestro punto de vista, es la autonomía tecnológica impulsada por los medios digitales la que experimentará un notable crecimiento en el futuro.
\end{abstract}

\section{Autonomía - Educación a distancia - tecnología}

ABSTRACT: This paper analyses the demand of autonomy and independence in learning as a decisive factor in the students' decision of taking regular courses in higher distance education. First, we have to separae independent learning, usual concept in (he specialized literature, and (he demand of au(onomy, as a motivation or, only, the answer to a question. This demand has recently grown and we suppose it will grow in size in the future. We have found the demand of autonomy differently spreads in (he students in he Universidad Nacional de Educación a Distancia (Spain). Next, demand of autonomy became technological autonomy: an autonomy without any classroom and presence of pro fessor. So, (he relation between pro fessor and sudent is reduced (o media and the moment of examination. In the future, this technologi cal autonomy will be specially pushed by the communication and information technologies.

\section{Autonomy - Distance Education -Technology}

\section{INTRODUCCIÓN}

La autonomía del estudiante es un rasgo que se ha atribuido a la enseñanza a distancia casi desde sus inicios, aun cuando no nace en su seno, sino en el de la denominada enseñanza programada. No obstante, ha quedado como parte intrínseca de la misma. Sim embargo y a pesar de los importantes acercamientos al respecto, quedan todavía numerosas características de este rasgo por explorar, desde las condiciones sociales para concretarlo como el esencial paso previo de su construcción teórica -pues como se pregunta Peters (1998): qué es un estudio autónomo-, hasta llegar, con el esfuerzo empírico, a observar su desarrollo en las distintas propuestas específicas de enseñanza a distancia.

El trabajo que aquí se presenta establece la concreción de la autonomía desde dos perspectivas. Desde el contexto espacial y temporal, se ubica en la Universidad Nacional de Educación a Distancia de España actual. Desde los sujetos de la autonomía, se sitúa en el ámbito de la demanda, es decir, en el peso que tal rasgo pudiera tener en la opción de estos estudiantes en favor de la enseñanza a distancia. Una doble concreción que, siendo necesaria, no deja de generar problemas, especialmente desde su formulación operativa:¿cómo preguntar sobre la demenda de autonomía? ¿cómo poner en duda la autonomía de los sujetos con una pregunta, en una sociedad que legitima la misma? La misma sociedad que produce enseñanza a distancia es la que produce autonomía. Por lo tanto, al hablar de demanda de automía habrá que tener buen cuidado en enviar las proyecciones individualistas, atribuyendo el cambio a nuevas generaciones de sujetos individuales. 
Dentro de un marco de evolución de la demanda de autonomía, cabe pensar que la misma adquiere aspectos distintos en función de las condiciones sociales y materiales en las que se desarrolla. Así, puede pensarse en una autonomía que hace hincapié en los procesos de evaluación, en otra que lo hace en los procesos de interacción entre docentes y alumnos u otra que simplemente destaca la menor subordinación de los alumnos a espacios y tiempos para seguir sus estudios. Como puede observarse , las tres especies de autonomía ejemplificadas se encuentran jerárquicamente articuladas. Pero, la diversidad de concreciones es amplia. Son diversos los grados de configuración del aprendizaje autónomo. El presente trabajo se centrará en un tipo de autonomía que aparece, en principio, más relacionada con las condiciones materiales, como es la disponibilidad de medios técnicos, que con las condiciones sociales, inclinándose auna mayor demenda de autonomía de los individuos.

\section{LA AUTONOMÍA Y EL APRENDIZAJE}

La procedencia griega del término autonomía conduce a darle el significado de la capacidad de imponerse la propia ley. Concepción extraña, inserta en una especie de reflexividad clausurada, por la que los individuos hacen constar su escisión del entorno. De hecho, sin referencia al entorno (físico, biológico o social), la autonomía es poco compresible. Así, la autonomía no es sólo el ejercicio de la propia ley. Es, sobre todo, la separacion de las otras lees de las leles de lo otros, implicando de esta manera, aun cuando sea indirectamente, una telacion causal entre tal entorno el sistema que se empieza a considerar autonomo. l), de la ausencia de autonomía, es el entorno el que causa el sistema. Desde su presencia, ha de reconocerse una inversion de la direccion de la causalidad, desde . 1 sistema hacia el entorno. Un sistema actua en el entorno.

La autonomía se comprende corno negación de la heteronomía. Por lo tanto, se obtiene del concepto una doble cara: la imposición sobre uno mismo, sobre una entidad que se constituye en identidad, y la ruptura con entidades ajenas de un nivel superior, ya sea un ni el lógico o sistérnico (entorno), ya sea un nivel jerárquico, como ocurre en el ámbito de la política y la sociedad. De aqui su sinculación COfl el concepto de independencia: ruptura con lo que produce dependencia y, por lo tanto, va sea desde la perspectiva lógica o social, subordinacion. Como señala Weber para el ámbito de la asociacion: «autonomía significa, al contrario que heteronomía, que el orden de la asociación no este otoqado -unpucsiopor alguien fuera de la inisnia y exterior a ella, suio por sus propias nuenilro,s y cii virtud de la cualidad de tales» (1979:40).

Desde la sociología, la autonomía es siempre algo relativo. Existe en dilerentes grados, como señala el mismo Weher (1 979:55). De aquí que no se pueda hablar de autonomía relativa, corno se autocorrige Luhmann (1996), sin alcanzar el pleonasmo. La autonomía es: «una variable que se encuentra entre la dependencia completa y el aislamiento completo del entorno)> (Luhmann, 1996:209).

Importa aquí aterrizar en los procesos del aprendizaje y del conocimiento. Si se puede reducir la autonomía a acto, es, sobre todo, un acto de reconocimiento, de constitución de una identidad, escindiéndose -utilizando el concepto preferido por la biología (Varela, 1979), en general, la denominada cihernetica de segundo orden (Morin, 1983:125 ss)- de un nivel-entidad superior y, a la vez, adscribiéndose a tal nivel-entidad: se es del mismo nivel que el entorno-origen-fuente de causalidad. En relación con el saber, la autonomía es, en principio, un saberse, un cobrar identidad y, desde aquí, un aprendizaje de sí mismo. Ahora bien, en la medida en que la nueva identidad en constitución se separa de la identidad-origen (entorno, a partir de tal constitución), queda dispuesta a nuevos aprendizajes, tanto de sí mismo, como del entorno. Por lo tanto, se amplia el proceso de autonomización: «El aprendizaje, concebido como conjunción de reconocimiento u descubrimiento, es un ejercicio de aulonomizacióli» (Morin, 1988:70). La autonomía se encuentra en el origen y en el resultado del aprendizaje. Es más, puede decirse que, estando en el origen adelanta parte del resultado; pero que no puede hablarse de resultado del aprendizaje si no ha existido un proceso de autonornización, puesto que no habría habido aprendizaje. En este proceso tendente al infinito, aprender es, sobre todo, aprender a aprender (Bateson, 1990). Afirmación que, en la deriva hacia procesos más específicos, plantea la mayor adecuación de modelos de enseñanza que implican centralmente tal autonomía, siendo conscientes de la exigencia del inicial reconocimiento autónomo de los receptores de la enseñanza. Doble ventaja comparativa de la enseñanza a distancia, pues requiere un lugar central de la autonomía en sus modelos pedagógicos y exige un previo reconocimiento de autonomía por parte de los alumnos. Las instituciones de enseñanza a distancia gestionan autonomía. Los alumnos de la enseñanza a distancia dan y demandan autonomía en el proceso de aprendizaje. La enseñanza a distancia no sólo produce sujetos sino que, en mayor medida que 
las tradicionales instituciones de enseñanza, necesita sujetos ${ }^{1}$. La idea de un alumno reducido al papel de pasivo receptor es más que discutible en todo proceso de enseñanza. Menos en el caso de la enseñanza a distancia.

El relativo nivel de abstracción usado a la hora de enfocar el concepto, no puede hacer olvidar su vinculación con procesos concretos, tanto históricos, como materiales y técnicos. Hablar de autonomía, al menos tal como se entiende hoy, pasa por su inscripción en la modernidad. Apenas cabe la autonomía, especialmente subjetiva, en la sociedad estamental (Weber, 1979:232 ss.). Tampoco puede dejarse a un lado su relación con prácticas y, lo que especial importancia para este trabajo, tecnologías para la autonomía. Norbert Elias (1987) realiza una brillante radiograña de la articulación de distintas prácticas, que se encuentran en la sociogénesis y psicogénesis de la autonomía y el individuo, en el paso de la sociedad feudal a la moderna sociedad cortesana. La imposición de la autonomía con el espíritu ilustrado va ligada a la tecnología (Luhmann 1996:339), entendiendo ésta, como se va a hacer a lo largo del artículo, de una manera amplia. Tecnología para la salvación autónoma (protestantismo), para el saber autónomo enfrentado a la autoridad excluyente de la fe y el dogma (procedimientos científico-metódicos), para la psique (Giddens, 1991:50-64) y el cuerpo (Outram, 1989; Duby, 1993; Giddens, 1995; Turner, 1996).

Sin entrar en lo que parece haberse convertido en el problema fundamental de la sociología que entra en el siglo XXI (Giddens, 1995:9), la cuestión de la modernidad, ha de subrayarse que ni la modernización se agota en la autonomización (Beck, 1997:41), ni la autonomización en la modernización. Ambos elementos se encuentran históricamente relacionados, apareciendo la autonomía como uno de los rasgos en la combinatoria del desarrollo de las sociedades, frente a las no desarrolladas (Peyrefitte, 1997:26). Es más, la articulación de desarrollo tecnológico y reflexividad institucional (Giddens, 1997) aparecen como un contexto aceleradamente autonomizante.

1 Con el concepto de sujetos, indisoluble del de aprendizaje, nos separamos de la concepción de autonomía de la teoría general de sistemas, donde alcanza un papel tan clave como autista (Beck 1997:41).

De vuelta al ámbito del aprendizaje, Descartes, a quien cabe otorgar alguna paternidad en el pensar moderno, estableció el círculo entre autonomía y conocimiento. Su Discurso del método se presenta como el logro autónomo, fruto de la experiencia, de un método para el conocimiento autónomo. De aquí que se ofrezca más como experiencia ejemplar, que como norma2. Una experiencia del conocimiento para el conocimiento, cuyo aprendizaje se reserva para el autónomo y colectivo lector de su libro.

\section{AUTONOMÍA Y EDUCACIÓN A DISTANCIA}

La relación entre educación superior a distancia y el concepto de autonomía es estrecha. Cabe recordar las palabras del profesor Carlos Moya, en los iniciales pasos de la Universidad Nacional de Educación a Distancia (UNED), en las que se vinculaba este tipo de educación con el modelo individualista (y autónomo) de la ética protestante pasado por la sociedad de masas. La apuesta del alumno por la educación a distancia puede entenderse, en principio, motivada por un deseo de individualización del proceso de aprendizaje. Al menos, como el reconocimiento de la propia capacidad para relacionarse con el conocimiento. Si en la propuesta calvinista la práctica religiosa se situaba preferentemente en el autoexamen, en una especie de combate consigo mismo; en la enseñanza a distancia, la disciplina del conocimiento empieza con la autodisciplina: con una especie de reconocimiento de las capacidades suficientes (cognitivas y para el establecimiento de rutinas) para emprender unos estudios que, en principio, se inician en solitario. En definitiva, puede entenderse como un reconocimiento de la capacidad para el esfuerzo en solitario, autónomo e independiente.

El notable salto cualitativo en el reconocimiento de autonomía, que también es un deseo de autonomía, cambia las formas del aprendizaje. Como indica el profesor Moya: "El aprendizaje deja de estar basado en la internalización, más o menos carismática, del discurso verbal del profesor, para depender casi exclusivamente de técnicas pedagógicas más o menos codificadas y sofisticadas, y con ello el arte pedagógico tradicional se convierte en tecnología social educativa” (ICEUNED, 1978:5). La tecnología se inserta de una manera central en el aprendizaje 3. Una relación entre tecnología educativa, distinta a la del discurso directo y verbal del profesor, y autonomía que no ha hecho sino retroalimentarse 
continuamente desde un origen que cabe situar históricamente en la extensión de la imprenta4. Retroalimentación que es el contexto para, a su vez, el continuo desarrollo de la enseñanza a distancia.

2 Véase especialmente las primeras páginas del Discurso del método (Descartes 1983 [1637]).

3 Desde la pedagogía, todo es susceptible de convertirse en tecnología. Véase la visión crítica de Zinnecker (1972).

4 La autonomía y el individualismo como producto de la escritura y lo colectivo, frente a una oralidad colectiva, es uno de los principios de la escuela canadiense que agrupa a Innis (1971a, 1971b),

Conducidos a sus raíces, los conceptos de autonomía o independencia informan todo proceso de aprendizaje sea presencial o a distancia. Así, puede entenderse el resultado del aprendizaje como el proceso hacia la independencia de la tutela del maestro y, en cierta medida, de la materia que se ha aprendido. Aprendemos algo cuando nos independizamos de ese algo. Ahora bien, se desea hacer hincapié aquí en la independencia y autonomía en el proceso de aprendizaje. No tanto en su resultado. La apariencia contradictoria del enfoque puede amortiguarse si se diferencia entre autonomía directa del maestro o profesor y, en especial, del contacto físico con éste (clase presencial), y autonomía de la clase que se aprende. Desde tal diferencia, se establece la posibilidad de alcanzar la autonomía de la materia (aprendizaje) con independencia de la presencia física del enseñante. Es una de las bases de la enseñanza a distancia, en la medida que tiende a reducir los contactos directos (presenciales) entre docente y discente.

La distancia física se complementa o sustituye por otros medios, a los que de manera global puede darse el nombre de tecnologías y materiales de aprendizaje ajenos a la presencia de la figura del enseñante. De aquí que, en la medida en que se articula autonomía de la presencialidad del enseñante e importante apoyo en tecnologías y materiales ajenos a la presencia del profesor, quepa denominar a esta circunstancia de autonomía tecnológica. Es, como ocurre con el concepto autonomía, una autonomía relativa, que, siguiendo las anteriores palabras de Moya, puede entenderse también como dependencia tecnológica.

La relación entre educación a distancia y autonomía se ha convertido en uno de los puntos de debate principal entre los expertos en este tipo de enseñanza y es uno de los elementos subyacentes cuando se discute en clave de modelos. Así, quienes, como Otto Peters (1983), conciben la enseñanza a distancia como una especificación en el ámbito educativo del modelo industrial, tienden a acentuar la importancia de la necesidad de autonomía controlada, algo sólo parcialmente paradójico, en el proceso de aprendizaje del alumno. Podria resumirse esta posición, no sin cierto abandono de los necesarios matices, como aquella que defiende la autonomía del alumno en un marco institucional rígido de las relaciones entre profesores y alumnos: a partir de una fuerte planificación previa de materiales, pruebas, consultas, etc., subrayando la idea de control, el alumno se conduce autónomamente. De aquí que esta autonomía, en especial cuando queda bajo perspectivas conductistas, tienda a parecerse a la autonomía del autómata. Por otro lado, quienes, siguiendo la estela de la proyección de los modelos macrosocioeconómicos al ámbito educativo superior a distancia, postulan, pues hay una buena dosis de normatividad, la necesidad de acercarse al marco del postfordismo (articulación entre unidades de menor tamaño y, sobre todo, flexibilidad como concepto que frecuentemente parece adquirir las características de lo mágico), defienden principalmente la idea de que la autonomía del alumno y, sobre todo, del futuro licenciado, hay que producirla en el propio proceso educativo. Si los primeros conforman un sujeto autónomo y solitario desde el principio, los segundos mantienen la validez de la autonomía al final del proceso, proponiendo que durante el proceso educativo se debe formar en tal autonomía y, sobre todo, ofrecer un marco para su desarrollo no precontrolado. Si en un caso puede hablarse de autonomía programada; en el otro, de autonomía en programación. Parte de este debate puede verse en Moore (1983), Daniel y Marquis (1979), Holmberg (1987), Sewart, Keegan y Holmberg (1983), Paul (1999) o Evans y Nation (1999).

McLuhan (1972, 1973), Havekicj (1963), Ong (1982) y, más recientemente, a Kerckhove (1999). De especial interés paa el futuro, en cuanto incorpora los medios de comunicación digitales, son las aportaciones reflexivas del último. Por otro lado y en el ámbito de este trabajo, que ya se ha referido al calvinismo, no debe olvidarse las relaciones entre protestantismo e imprenta. 
La autonomía es un concepto admitido por casi todos, derivado de su carga positiva en los procesos de comunicación educativa. Como señala Paul, haciendo depender la consecución final de la autonomía del alumno del papel del profesor y de la universidad, se trata de un ideal: "Si el alumno ideal es aquel que a la larga supera a su profesor, la universidad abierta ideal es aquella que engendra alumnos que no precisan ya de tutela" (Paul, 1999:44).

Como ya se habrá percibido y a pesar del esfuerzo del primer apartado de este artículo, uno de los problemas es de qué hablamos cuando se invoca el término autonomía., pues puede ser entendida de muy distintas maneras. Múltiples significados para un término que cubre un campo semántico tal vez demasiado amplio como para seguir siendo útil. No obstante, en buena parte de los mensajes publicitarios y propagandísticos, si es que en nuestros días de mezcla de lo político y lo comercial pueden separarse ambos calificativos, de las universidades a distancia se afianza la idea de lo que puede denominarse autonomía tecnológica: el alumno puede desarrollar sus estudios cómoda y fácilmente en su casa, a partir de las tecnologías digitales de la información (correo electrónico, Internet, materiales multimedia, televisión digital). Tal autonomía tecnológica, especialmente abanderada por las ofertas de educación universitaria a distancia de nueva creación, tiene como principal característica la eliminación de la relación personal, en clase, con el profesor e incluso con el profesor-tutor. La relación siempre es mediada a través de un soporte técnico.

El auto-aprendizaje a través de tecnologías está presente en los principales expertos de la enseñanza a distancia. Así, cabe destacar la figura de Rowntree (1990), aun cuando su tecnología es fundamentalmente la escritura y sus consejos vayan dirigidos a la redacción de materiales escritos. Lo que incorporan las nuevas tecnologías de la información es cierta interactividad a tal auto-aprendizaje. El alumno podría lanzar sus mensajes de solicitud de tutoría a cualquier hora y en cualquier día. Por ejemplo, el correo electrónico o los grupos de discusión en la red permitirían una especie de "comunicación continua"; al menos, la creencia en un vínculo constante entre docentes y alumnos, aparentemente no muy distinto de los servicios " 24 horas" ofrecidos por bancos, aseguradoras y otros tipos de empresas. Es decir, la flexibilidad se incorpora al momento de hacer las consultas. A la autonomía-autómata del alumno se añade la autonomía de la disposición del tiempo de consultas. Desde la sociología, recientemente se ha subrayado la importancia que tiene el tiempo en la estructuración de la sociedad (Giddens 1991) y, por lo tanto, de las relaciones sociales, lo que incluye las relaciones entre profesores y alumnos. Con "viejas” o "nuevas” tecnologías, se está defendiendo la idea de que el proceso de la enseñanza universitaria a distancia se apoya básicamente en un alumno autónomo, en el que apenas tiene papel la relación personal de carácter presencial con el profesorado.

No faltan opositores al concepto de autonomía en la enseñanza a distancia. Desde la asimilación de autonomía tecnológica y dependencia tecnológica, que, en el fondo, sigue tomando como modelo ideal el de la enseñanza presencial, a las razonables críticas político-ideológicas, como la de Paul, para quien el "alumno autónomo" es un mito. Según este autor, la autonomía del alumno ha de ser un objetivo y no un punto de partida. Por otro lado, la ubicación de la autonomía en el inicio del aprendizaje puede esconder modelos autoritario-tecnocráticos o, al menos, poco participativos, a pesar de hablarse de interactividad. La necesidad de los alumnos para ponerse en contacto entre sí, de contarse sus problemas, incluso la posibilidad de criticar a los profesores o la institución, parecen difícil en las comunicaciones a través de la red u otros medios distintos a los presenciales. Ha de tenerse en cuenta que si en el conjunto de las comunicaciones personales cara a cara, cabe fácilmente la sectorización o privatización de una parte de los mismos, las comunicaciones digitales son públicas y, en cierta forma, bajo control, hasta en los más participativos grupos de discusión: ¿cabría en ellas la crítica a la forma de programar la asignatura o al profesor, o, simplemente, la exposición pública de las propias deficiencias, algo parecido a un "no entiendo nada de lo que se me dice en esta asignatura porque está mal diseñada su enseñanza”?.

Si se mira sólo pocos años atrás, hablar de la enseñanza superior a distancia en España como una opción derivada de una motivación - deseo de autonomía- puede carecer de sentido para la mayor parte de los casos. Es cierto que una parte importante del alumnado inicial de la UNED presentaba un perfil sociodemográfico y actitudinal fruto de la modernización y racionalización del país: hincionarios con importante motivación hacia el logro y la autosuperación, en buena parte procedentes de clases populares y en proceso ascendente ${ }^{5}$. El afán de superación parecía arraigado y, en buena parte, explica la masiva demanda de estudios superiores a la que la UNED intentaba dar respuesta. Sin embargo, su opción por el modelo de la enseñanza a distancia tendía a derivar más de condiciones no elegidas, como la falta de tiempo disponible para asistir a las aulas o la carencia de oferta de enseñanza universitaria en la localidad 
de residencia, que de una selección a favor de la autonomía personal en el proceso de aprendizaje. De hecho, tal disociación se establece como marco para la extensión de unos mediadores claves en el modelo de la UNED: los centros asociados y los tutores de asignaturas. Fundamentalmente se trataba de dar oportunidades y canales para dar salida a una demanda de estudios universitarios que no podía satisfacer el sistema universitario español; pero desde el mantenimiento de que el modelo ideal era el aportado por la enseñanza presencial.

5 En el estudio dirigido por el profesor Santos Juliá sobre los primeros mil licenciados de la UNED se decía: "por ocupación, por nivel de estudios, por dimensiones de hábitat, el licenciado de la UNED es una persona que se encuentra, cuando decide matricularse en esta Universidad, en un proceso de movilidad social ascendente y que tiene conciencia de no haber llegado al límite de sus posibilidades de ascenso: una buena parte de ellos confiera que el principal motivo que les llevó a matricularse en la Universidad fue su promoción profesional. Habiendo ascendido algunos peldaños en la escala social, con una titulación de bachiller o de grado medio, la licenciatura se ofrece como coronamiento de un proceso ya iniciado, como representación simbólica de ese proceso, a la vez que como recurso para un potencial nuevo paso hacia delante” (UNED, 1981).

Los más de veinticinco años transcurridos desde la fundación de la UNED han transformado notablemente el país. Importa aquí resaltar dos aspectos: a) la extensión de la oferta universitaria presencial, llegando muchas localidades de las que se encontraba ausente; b) la creciente configuración de la enseñanza a distancia como una alternativa más, abandonando sus pesadas connotaciones negativas como enseñanza de "segundo orden", sobre todo a partir de la rápida implantación de las nuevas tecnologías de la información y la comunicación. Aspectos que alimentan la suposición de que la enseñanza universitaria es hoy, al menos más que ayer, una opción y no una solución a condiciones exógenas. Una vez cubiertas algunas de sus deficiencias por el sistema de educación superior presencial, como es la extensión geográfica de su oferta, es cuando puede empezar a plantearse que la opción por la enseñanza superior a distancia entra en la órbita de la opción. Entonces, es posible dar algunos pasos desde el discurso de la compensación de las condiciones desfavorables o el del fomento de la igualdad de oportunidades al discurso de la opción por la autonomía.

A pesar de que la autonomía en el proceso educativo tal vez sea un mito, ha de reconocerse que está muy presente en la imagen que tiene la sociedad, en general, y de los estudiantes de la UNED, en particular, del tipo de enseñanza que se ofrece en las universidades a distancia. Así, un 76,8\%6 de los alumnos matriculados en la UNED en el curso 1997/98 mantienen que ha influido mucho o bastante la independencia y autonomía que permiten la enseñanza a distancia para cursar estudios en esta universidad. Pues bien, se analizará sintéticamente: a) las características de esta demanda general de independencia y autonomía; b) la relación entre tal demanda general y la específica demanda de autonomía tecnológica; y c) el perfil de quienes parecen optar por esta autonomía tecnológica como principal lazo de relación en el proceso docente. Los datos se toman fundamentalmente de la encuesta del curso 1997/98 ya referida, con las relativas limitaciones derivadas de que la respuesta a tales cuestiones no contaba como una de sus principales finalidades.

6 Encuesta mediante cuestionario autoaplicado que se incluye en el sobre de matrícula del curso 1997/98, de la que se extrajo, para su análisis, una muestra aleatoria de 8009 alumnos. La encuesta fue llevada a cabo por el IUED (UNED).

\section{METODOLOGÍA}

Los datos que sirven de referencia forman parte de los resultados de la en- cuesta que rellenaron los alumnos en el momento de su matriculación para el curso 1997-1998. Se trata, por lo tanto, de un cuestionario autoaplicado, en el que la opción de completarlo era voluntaria en todo momento, garantizándose en su inicio la confidencialidad y resguardo de las respuestas individuales y, en definitiva, la adscripción al código deontológico de la investigación social, que impide todo uso de la información recogida, más allá del tratamiento estadístico de los datos acumulados.

De todos los cuestionarios retornados en el propio sobre de matrícula, se obtuvo aleatoriamente una muestra estratificada por carreras, tomando los matriculados en el Curso de Acceso como una carrera más. El tamaño definitivo de la muestra fue de 8.009 cuestionarios, de los cuales 7.274 corresponden a 
cuestionarios válidos de individuos que se matricularon en una carrera universitaria, dejando a un lado los matriculados en el Curso de Acceso. Debe tenerse en cuenta que el total de alumnos matriculados en las distintas carreras universitarias de enseñanza reglada de la UNED del año académico referido fue de 122.322 individuos.

El cuestionario contó con 43 preguntas, aun cuando más de un tercio de las mismas estaban dirigidas sólo a los alumnos antiguos, que componían el 72,3\% de la muestra final, con inclusión del Curso de Acceso.

Para establecer el grado de independencia se tuvieron especialmente en cuenta las preguntas relativas a la razón de matriculación en la UNED (p.16), distintos aspectos que pueden servir de ayuda para el estudio (p.20) y la consideración de los aspectos que en mayor medida pueden influir en el aprovechamiento del estudio.

\section{CARACTERÍSTICAS DE LA DEMANDA DE AUTONOMÍA}

En un 76,8\% de los cuestionarios analizados se señala que la independencia y autonomía que permiten la enseñanza a distancia ha influido mucho o bastante para cursar estudios en la UNED. Entre los motivos expuestos, es el más apoyado, como muestra la siguiente tabla:

\begin{tabular}{|c|c|c|c|}
\hline & $\begin{array}{l}\text { Total cuestionarios } \\
\text { válidos }\end{array}$ & $\begin{array}{l}\text { Mucho/ } \\
\text { Bastante }\end{array}$ & $\begin{array}{l}\text { Poco/ } \\
\text { Nada }\end{array}$ \\
\hline $\begin{array}{l}\text { Por la independencia y autonomia que } \\
\text { permiten la enseñanza a distancia }\end{array}$ & 7.461 & $76,8 \%$ & $23,2 \%$ \\
\hline $\begin{array}{l}\text { Por la posibilidad de compaginar los } \\
\text { estudios con obligaciones familiares }\end{array}$ & 7.466 & $60.9 \%$ & $39,2 \%$ \\
\hline $\begin{array}{l}\text { Por existir un centro de la UNED en mi } \\
\text { localidad o lugar de trabajo }\end{array}$ & 7.214 & $41,8 \%$ & $58.2 \%$ \\
\hline $\begin{array}{l}\text { Porque en mi localidad no hay Univer: } \\
\text { sidad }\end{array}$ & 7.202 & $27,7 \%$ & $72,3 \%$ \\
\hline $\begin{array}{l}\text { Porque no ha podido acceder a otra } \\
\text { Universidad }\end{array}$ & 7.205 & $36,8 \%$ & $77,4 \%$ \\
\hline
\end{tabular}

A la vista de este resultado, cabe concluir que una de las principales razones del notable crecimiento de alumnos matriculados en esta universidad reside en la independencia y autonomía que procura para desarrollar los estudios. No existe posibilidad de comparación directa con resultados de encuestas de años anteriores, pero algunos indicios parecen señalar el importante salto cualitativo dado. Así, en la encuesta a los matriculados en el curso 1980/8 1, la ventaja de la UNED señalada en primer lugar por una mayor proporción de encuestados (45\%) era la de llegar a los que no pueden estudiar por dificultades económicas y de desplazamiento. La segunda ventaja señalada, con un $19 \%$ de las manifestaciones ya se acerca algo más a la idea de autonomía: "Permite un horario flexible de estudio" (ICE-UNED, 1982). La conclusión dista de ser definitiva, pero parece haber indicios de que, en estos casi 20 años transcurridos, se ha pasado de una concepción de esta enseñanza superior a distancia como cobertura de la relativamente escasa extensión del sistema universitario presencial español, a la de una alternativa claramente diferencial caracterizada por facilitar mayores cotas de independencia y autonomía.

Tal deseo de independencia y autonomía no está homogéneamente distribuido entre todo el alumnado; pero tampoco pueden considerarse las diferencias como excesivas. La edad aparece como una variable significativa al respecto, con una relación positiva: a mayor edad, mayor es la proporción de entrevistados que optan por la independencia y autonomía: la media de edad entre los que dicen que en la decisión de estudiar en la UNED ha influido mucho este factor está en 31,3 años, para los que señalan la opción "bastante" en 30,88 años y así la media de edad de 28,44 años para los que han manifestado que no ha 
influido nada. La opción por la independencia y autonomía aparece relacionada con el proceso de maduración personal vinculado a la edad.

La opción por la independencia y la autonomía puede venir dada por las propias condiciones contextuales de los individuos, de tal manera que si tienen otras ocupaciones, especialmente de carácter laboral, la inclinación por la misma tiene más un carácter de necesidad que de opción. Pues bien, las diferencias en hinción de la situación laboral existen, como muestra el siguiente cuadro:

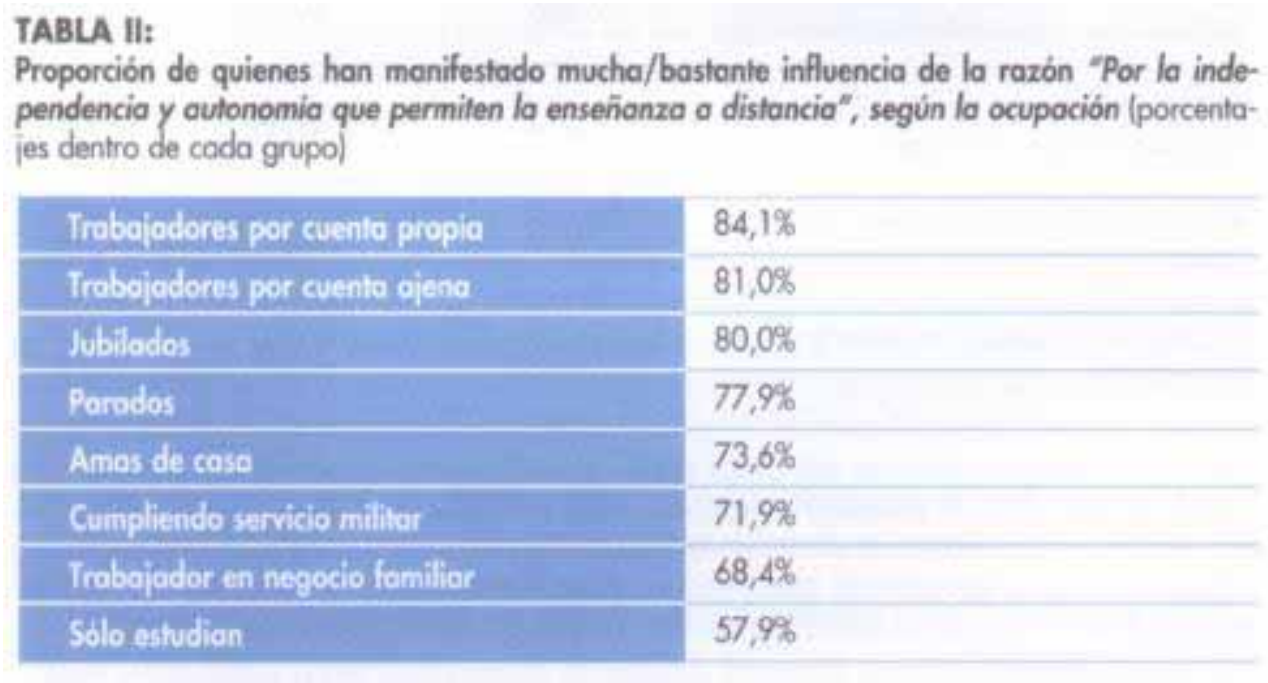

Las diferencias entre unas situaciones laborales y otras son relevantes: más de 26 puntos porcentuales entre quienes trabajan por cuenta propia y quienes sólo estudian. Sin embargo, ha de resaltarse la propia mediación de la edad (los más jóvenes se encuentran entre quienes sólo estudian) y que en todas las situaciones laborales, incluso aquellas como la de quienes sólo se dedican a estudiar, son mayoría los que señalan la influencia de la autonomía y la independencia para matricularse en la enseñanza a distancia. Es más, entre estos sigue siendo la principal razón, seguida con un $44,6 \%$ de quienes señalan mucha/bastante influencia del hecho de no haber podido acceder a otra Universidad.

Si se atiende a las carreras que se cursan, es Filosofía la que mayor proporción de matriculados con este deseo presenta (86,1\%), mientras que Químicas $(53,4 \%)$ e Ingeniería Industrial $(57,9 \%)$ las de menores proporciones, lo que podría llevar a una diferenciación entre Letras y Ciencias de manera que aparentemente es la característica de la materia que se aprende la que permite una mayor o menor independencia y autonomía del alumno. De esta manera, cabría señalar que unas carreras y disciplinas (Letras y Sociales) tienen más facilidad de adaptarse a un modelo de educación superior a distancia regido por la autonomía del alumno; mientras que otras (Químicas, Ingeniería, Físicas) deben estructurarse sobre un modelo que otorga menos autonomía, ofreciendo mayor apoyo. Ahora bien, ha de tenerse en cuenta la mediación de la variable edad, puesto que carreras universitarias que presentan las mayores proporciones de demandantes de autonomía, como Derecho, Geografía e Historia, Filología o Filosofía son las que tienen una medias de edad de su alumnado mayores: 33,30 años, 33,42 años, 32 y 31,71 años respectivamente. Sin embargo, son precisamente Químicas e Ingeniería Industrial las que tienen medias de edad más bajas: 25,2 años y 26,12 años respectivamente.

Como suele ocurrir en ciencias sociales, hablar de relación causal entre una variable otra es arriesgado. Así, optar por la afirmación de que los más adultos se inclinan por carreras que permiten más autonomía, como si este deseo fuese previo a la elección de la carrera, o por la de que tal sector de los estudiantes eligen las carreras por no disponer de las capacidades y tiempo de otras carreras menos autónomas, no puede dirimirse aquí. Entre la elección y la adaptación de la elección a las circunstancias, como adaptación a la propia posición social (Bourdieu, 1988), hay una frontera de difícil identificación.

Al existir relación entre la edad y la demanda de autonomía e independencia, parece lógico pensar que también la hay con respecto al curso en el que se matriculan los alumnos. Sin embargo, la siguiente tabla ofrece un obstáculo a tal expectativa: 


\begin{tabular}{|c|c|c|}
\hline $\begin{array}{l}\text { Curso } \\
\text { matriculado }\end{array}$ & $\begin{array}{l}\text { Porcentajo mantienen mucha/bastante } \\
\text { influencia de outonomia e independencia }\end{array}$ & $\begin{array}{l}\text { Medio de edod do alumnos } \\
\text { matriculados en chîos }\end{array}$ \\
\hline Primero & $77,8 \%$ & 29,22 \\
\hline Segundo & $72,1 \%$ & 30,26 \\
\hline Tercero & $74,0 \%$ & 31,58 \\
\hline Cuarto & $76,8 \%$ & 32,33 \\
\hline Quinto & $80,3 \%$ & 34,02 \\
\hline
\end{tabular}

Hay un mayor deseo de autonomía e independencia entre los alumnos que se matriculan en el primer curso, que entre los de segundo, tercer y cuarto curso. Después, se aprecia una notable recuperación en el quinto curso. Son diversas las interpretaciones que pueden derivarse de estas distribuciones.

Por un lado, al descender notablemente la demanda de autonomía e independencia desde los alumnos matriculados en el primer curso a los matriculados en el segundo, puede entenderse por el hecho de que un sector se enfrenta a la dura faceta de la autonomía y la independencia, disminuyendo sus demandas en el segundo año, o porque la independencia y autonomía demandada de este sector no se corresponde con la ofrecida por la Universidad. Esta última interpretación aparece parcialmente avalada por el dato de que se ven influidos en mayor proporción por la independencia y la autonomía los alumnos que se matriculan por primera vez (80\% señalan verse influidos mucho o bastante por esta razón en su opción por la UNED); mientras que entre los alumnos que ya se habían matriculado en años anteriores el porcentaje desciende hasta el $75,4 \%$. Por otro lado, la evolución que toma esta demanda a lo largo de los cursos y, sobre todo, el salto dado desde el cuarto al quinto curso, parecen mostrar que el propio paso por la institución socializa en la independencia y la autonomía, haciéndolo uno de sus principales valores en la vinculación con la UNED; pero aquí nos encontramos nuevamente con la mediación de la edad: ¿es la maduración en la institución o, simplemente, la maduración en función de la edad, del paso vital, la que lleva a una mayor demanda de autonomía? Un sencillo índice puede ayudar a resolver parcialmente la resolución a favor de una u otra vía de interpretación de estos resultados. En la siguiente tabla, se muestra la relación entre el porcentaje de los alumnos que mantuvieron en la encuesta que se inclinaron por este tipo de enseñanza por la autonomía y la independencia y, en el denominador, la edad media en cada uno de los cursos:

TABLA IV:

Indices de peso de la autonomía e independencia en función de la edad por curso de carrera universitaria matriculado

\begin{tabular}{l|c|}
\hline $\begin{array}{l}\text { Curso matriculado. } \\
\text { Porcentaje mantienen mucho/bostante influencio de outonomia } \\
\text { e inclependencia en función de la medio de edod de oluminos } \\
\text { matriculados en años }\end{array}$ \\
\hline Primero & 2,66 \\
\hline Segundo & 2,38 \\
\hline Tercero & 2,34 \\
\hline Cuerto & 2,37 \\
\hline Quinto & 2,36 \\
\hline
\end{tabular}

Se observa cómo, teniendo como elemento mediador la edad, sigue siendo el primer curso el que presenta mayor peso de la demanda de autonomía e independencia como factor a la hora de matricularse en la UNED. Sin embargo, en los otros cursos, el índice elaborado es bastante semejante. Es decir, existe una demanda de autonomía e independencia por parte del nuevo alumnado en la institución que ésta no parece satisfacer convenientemente o a la que una proporción de los alumnos no es capaz de adaptarse. 
La disminución relativa de la demanda de autonomía e independencia desde el primer curso a los otros cabe atribuirla tanto un reconocimiento de insuficientes capacidades para desarrollarla, por parte de un sector del alumnado, como al reconocimiento de éste del hecho de que la institución no tiene en sus rasgos tal facilitación de la autonomía y la independencia, de manera que no puede ser un factor que motive a seguir matriculándose en la institución. Esta segunda explicación parece más alejada de la realidad, creyendo conveniente inclinarse por la aceptación de la primera, por la que el alumno siente que puede emprender un proceso de aprendizaje de manera autónoma e independiente de la asistencia a clases presenciales, enfrentándose, una vez matriculado, con el insalvable esfuerzo que le supone tal tipo de enseñanza. Esto explicaría el constante y notable descenso de matrícula desde los primeros cursos de las carreras a los siguientes y, sobre todo, la relativamente alta proporción de los nuevos alumnos de los que apenas se tiene constancia de más actividad que de la propia matriculación, ya que no se presentan a examen. Así, aproximadamente el 55\% de los alumnos matriculados en las asignaturas de los primeros cursos de carrera figura como no presentado, variando de una carrera a otra (IUED 1998).

Así, nos acercamos a una de las diferencias más relevantes: si el deseo de autonomía e independencia alcanza al 80\% de los alumnos que se matriculan por primera vez en la UNED, sólo llega al 75,4\% de los que son antiguos alumnos. Un descenso que, en función de lo visto, cabe achacarlo al hecho de que una parte de estos alumnos que desean independencia y autonomía se ven incapaces de llevarla a cabo y no vuelven a matricularse.

\section{CARACTERÍSTICAS DE LA AUTONOMÍA DEMANDADA}

Claro está, autonomía y posibilidad de apoyo en el desarrollo del proceso de aprendizaje no son incompatibles. Es más, lo deseable es su articulación. Pero, en principio, parece que en la medida en que se ofrece mayor cantidad y diversidad de apoyos a la enseñanza, cada uno tiende a introducir cierta rigidez. Piénsese en las clases presenciales u oferta de tutores presenciales, que introducen rigideces de carácter espacial - hay que señalar un lugar de encuentro- y temporal, pues el encuentro se establece en un horario. Desde tal argumento, puede asumirse que la ventaja comparativa de las aportación de las tecnologías de la información y la comunicación en la educación es su relativa flexibilidad. Por lo tanto, la autonomía parece relacionarse en mayor medida con tales soportes técnicos.

Como aproximación a las características que tiene esta demanda de autonomía, parece conveniente acercarse a qué tipo de demandas incluye esta demanda de autonomía. Para ello, se cruzan los resultados de quienes han optado por las categoría mucho o bastante en la opción por la independencia y autonomía que permiten la enseñanza a distancia, como factor que ha influido en la decisión de cursar estudios en la UNED, con quienes han señalado las mismas categorías en la pregunta sobre la consideración de distintos apoyos para el estudio:

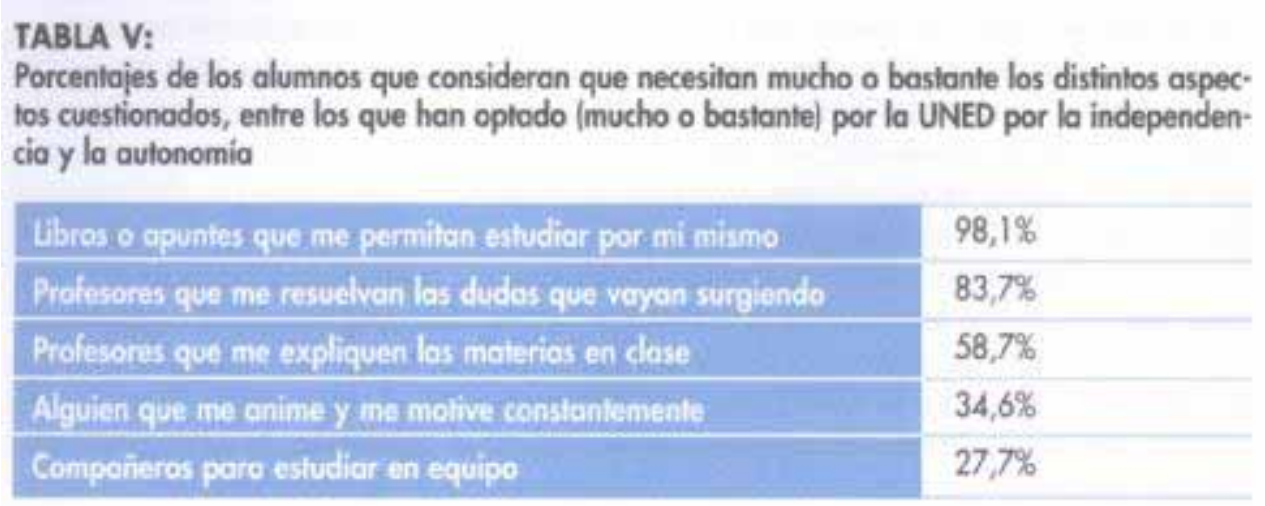

A partir de la tabla y, se observa que la autonomía pasa por los libros o apuntes y por la existencia de profesores que vayan resolviendo las dudas. Dos demandas que, en principio, cabe considerarlas como obvias, especialmente la primera. Es decir, la concreción de la autonomía exige de materiales producidos para tal autonomía. La autonomía del profesor se compensa con la dependencia de los materiales.

Un resultado algo más sorprendente lo constituye el relativo alto porcentaje de los que, teniendo la autonomía como determinante para su matriculación en la UNED, requieren la existencia de profesores 
que expliquen las materias en clase. un 58,7\%. Tal resultado nos indica que una parte sustancial de los alumnos no optan por una autonomía que prescinda de la presencia física del profesor.

No cabe duda de que este resultado ayala modelos de enseñanza a distancia que contemplan el contacto físico periódico entre profesores y alumnos. No obstante, antes de llegar a tal afirmación ha de hacerse una mínima reflexión metodológica: a) la muestra está formada por alumnos de la UNED, de los que se puede suponer que, en buena parte, eligieron esta opción por el propio modelo de enseñanza a distancia que ofrece esta institución, que incluye la presencia de profesores tutores en los Centros Asociados (centros de carácter local distribuidos en distintos puntos geográficos, tanto en España, donde se encuentran la mayoría, como en el extranjero); b) tal como está realizada la pregunta en el cuestionario donde las alternativas se reducen al grado en que se consideran necesarios "profesores que me expliquen las materias en clase"- se tiende a favorecer la "demanda”, es decir, a que quien responda se incline por solicitar indirectamente en sus respuestas el mayor número de apoyos, especialmente de cara a la institución. Con relación a este último punto, el 57,3\% de los encuestados han contestado con las opciones mucho o bastante a los tres primeros aspectos señalados en la tabla V: libros, profesores para dudas y clases.

El análisis de las características de la autonomía demandada nos lleva a reconocer el importante peso de las clases presenciales. Ahora bien, desde la casi identificación entre demanda de autonomía e independencia y necesidad de materiales (tecnologías, entendiendo el concepto en un sentido amplio), puede considerarse como autonomía tecnológica la demandada por aquellos que han mantenido en el cuestionario que no necesitan para seguir sus estudios las clases presenciales. Por lo tanto, se entiende como autonomía tecnológica la que no desea pasar por el aula presencial. Así, puede concluirse que un 31,5\% de quienes respondieron a la encuesta analizada demanda tal autonomía tecnológica.

Ha de anotarse que tal proporción ha de considerarse como demanda fuerte o mínima, en cuanto excluye el paso por el aula. La percepción del porcentaje resultante como mínimo queda subrayada con la comparación con otras observaciones, como la realizada por Pérez Juste y colaboradores (1999), donde la demanda tecnológica queda enfrentada directamente a la demanda de clases o tutorías presenciales. En tal estudio, el porcentaje de alumnos encuestados7 que prefirió la tutoría a través de medios telemáticos sobre la tutoría presencial fue del 52,9\%, como muestra la siguiente tabla:

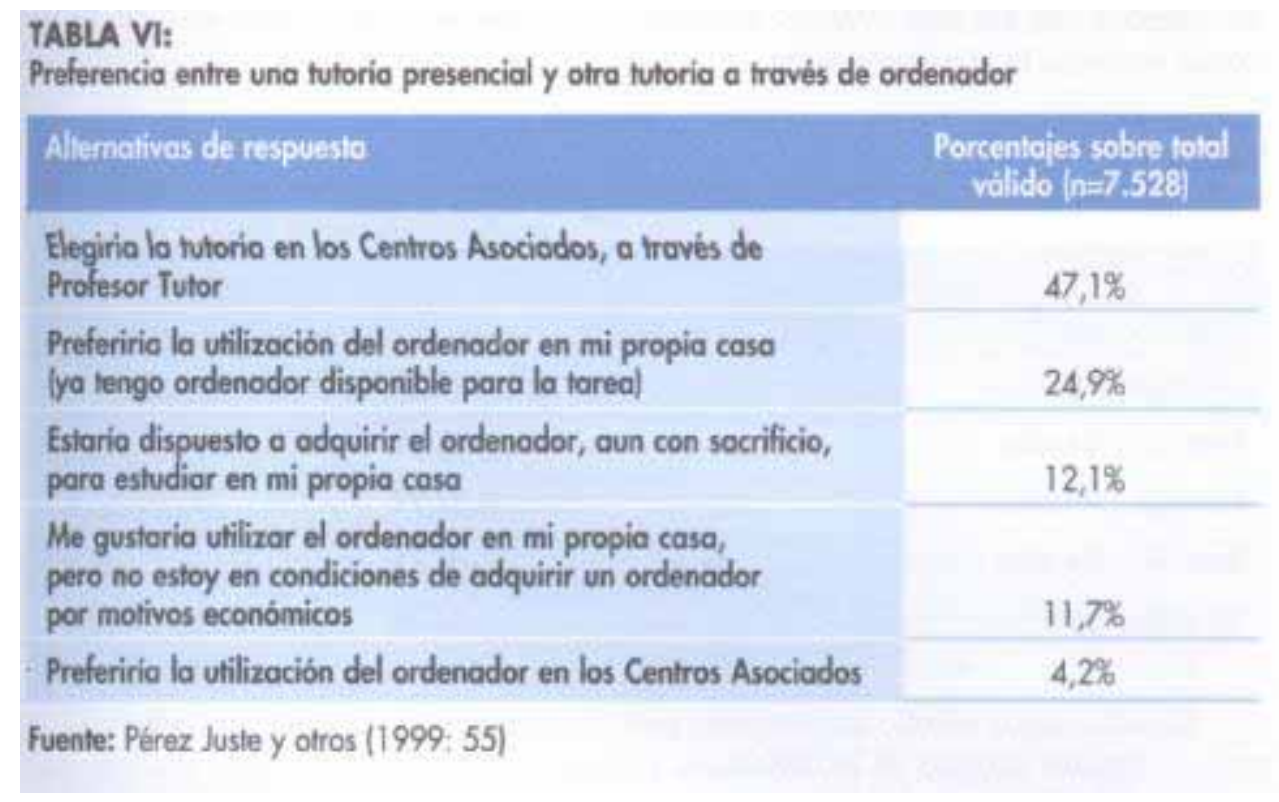

El porcentaje, del total de alumnos que contestaron a la pregunta, que prefiere la utilización del ordenador propio, en su casa, es 48,7\%, superando el de quienes optan por la tutoría presencial (47,1\%). Puede decirse que, en la actualidad, el conjunto de los estudiantes de la UNED se encuentra dividido por la mitad entre los dos modelos (clases, por un lado, y autonomía tecnológica, por otro), desde el supuesto de exclusión respectiva. Ahora bien, dado el fuerte crecimiento que en nuestra sociedad experimenta el uso del ordenador e Internet8, es previsible un aumento de la inclinación por la enseñanza a través de este medio en el futuro inmediato. 
7 Fue encuestado todo el alumnado de la UNED que decidió matricularse en una o más asignaturas durante el año académico 1997-98. Para más detalles, véase Pérez Juste y otros (1999).

\section{PERFIL SOCIAL DE LA DEMANDA DE AUTONOMÍA TECNOLÓGICA}

Antes se recogieron los criterios sociológicos principales que parecían condicionar la mayor presencia relativa del factor independencia y autonomía en la decisión de cursar estudios en la UNED. Ahora se trata de observar el perfil de quienes basan tal demanda en la posibilidad de prescindir de clases presenciales. Es decir, las características del 31,5\% de los alumnos señalado anteriormente.

La distribución de la autonomía tecnológica en función de la edad ya no aparece en un continuo, aun cuando siguen siendo más demandantes de la misma los adultos que los más jóvenes. En concreto, quienes tienen entre 35 y 54 años, como muestra la siguiente tabla:

\begin{tabular}{|c|c|}
\hline Intervalos de edad & $\begin{array}{l}\text { Porcentojes de demondantes de aulonomia lecnológica } \\
\qquad \text { en funcion de edod }\end{array}$ \\
\hline Menos de 24 años & $22,2 \%$ \\
\hline Entre 25 y 34 oños & $33,8 \%$ \\
\hline Entre 35 y 44 años & $34,9 \%$ \\
\hline Entre 45 y 54 años & $34,7 \%$ \\
\hline 55 o más años & $33,3 \%$ \\
\hline
\end{tabular}

La edad sigue siendo importante; pero aparecen otros relevantes factores, como el carácter urbano de la demanda, ya que la proporción se eleva a cifras por encima del 39\% entre los alumnos que residen en municipios entre 250.000 habitantes y un millón. Al cruzar la edad y la variable tamaño de hábitat, se observa que el 52,2\% de los alumnos entre 35 y 44 años, que viven en ciudades de más 000 habitantes y menos de un millón de habitantes, demandan autonomía tecnológica.

8 En cuatro años (1996-2000), la proporción de españoles de 14 o más años que tiene acceso a Internet ha pasado del 1,4\% al 12,4\%. Para más detalle, véase la encuesta del Estudio General de Medios (última referencia obtenida en abril del año 2000: www.aimc.es).

Asimismo, hay que destacar el $45,2 \%$ que alcanza entre los trabajadores por ita propia.. Si bien suponen una pequeña parte de la muestra (5,5\% de los en- tas), ha de destacarse que se trata de un sector en crecimiento en las socies desarrolladas y, en especial, en ámbitos económicos avanzados tecnológiente (asesores, profesionales, etc.).

La clase social, derivada de la pregunta sobre el nivel de estudios del padre, $\mathrm{n}$ condicionante, estando más extendida la demanda de autonotecnológica entre las clases sociales superiores del alumnado cuyas respueshan sido analizadas. Mientras el $41,9 \%$ de las encuestas de alumnos con padres que completaron estudios superiores demanda tal autonomía tecnológica, desciende al $27 \%$ entre cuyos padres no completaron ningún tipo de estudios.

Una de las características de esta demanda tecnológica debiera estar, supuestamente, en el uso de medios. Algo que puede observarse a partir de los resultados de las respuestas a la pregunta 39 en las encuestas analizadas, sobre la utilidad que ha tenido para los alumnos antiguos (matriculados en cursos anteriores en la UNED) distintos materiales didácticos. La distribución ha sido la siguiente: 


\begin{tabular}{|c|c|c|c|c|}
\hline Material Didactico & $\begin{array}{l}\text { Autonomia } \\
\text { fecnologica }\end{array}$ & $\begin{array}{l}\text { Autonomia } \\
\text { con clases }\end{array}$ & $\begin{array}{l}\text { No demanda } \\
\text { de aulonomio, } \\
\text { si clases: }\end{array}$ & $\begin{array}{l}\text { Ni autonomia, } \\
\text { ni dases }\end{array}$ \\
\hline Radio & $21,6 \%$ & $21,4 \%$ & $14,9 \%$ & $16,2 \%$ \\
\hline Audiocosetes & $8,4 \%$ & $11,3 \%$ & $8,6 \%$ & $7,2 \%$ \\
\hline Videos & $6,6 \%$ & $8,4 \%$ & $7,0 \%$ & $6,1 \%$ \\
\hline $\begin{array}{l}\text { Moterial informático } \\
\text { y mulhimedia }\end{array}$ & $9,1 \%$ & $10,7 \%$ & $8,0 \%$ & $6,1 \%$ \\
\hline Televisión & $6,0 \%$ & $7,0 \%$ & $5,7 \%$ & $3,3 \%$ \\
\hline $\begin{array}{l}\text { Correo electrónico, } \\
\text { video conferencias }\end{array}$ & $7,7 \%$ & $8,6 \%$ & $6,5 \%$ & $7,5 \%$ \\
\hline
\end{tabular}

Se observa en la tabla anterior que no son los demandantes de autonomía tecnológica los que más utilidad han encontrado en los distintos materiales didácticos-tecnológicos. De manera sistemática, han sido los alumnos que demandan autonomía y, a la vez, presencia de clases, los que han ido encontrando utilidad en tales materiales. Ahora bien, dentro de un contexto general en el que la mayoría de los alumnos han encontrado poca o ninguna utilidad a los mismos, con relativa excepción de la radio. Claro está, la pregunta no está referida tanto al uso de medios como a la utilidad encontrada, siendo ambos conceptos diferentes, pues el segundo se relaciona más directamente con una valoración de los medios. Así, podría entenderse que la menor utilidad encontrada por quienes demandan autonomía tecnológica se deba a que su exigencia de utilidad es mayor o a que realmente han utilizado poco tales medios, con lo que la autonomía tecnológica se basa fundamentalmente en libros y apuntes, material escrito, como parece mostrar la siguiente tabla:

\section{TABLA IX:}

Porcentajes de respuestas del grado de necesidad de libros o opuntes para llevar a cabo el estudio autónomamente, según la demanda de autonomia

\begin{tabular}{l|cccc|}
\hline $\begin{array}{l}\text { Grodo en que considera } \\
\text { necesario libros } \\
\text { y opuntes }\end{array}$ & $\begin{array}{c}\text { Autonomia } \\
\text { fecnologica }\end{array}$ & $\begin{array}{c}\text { Autonomia } \\
\text { con clases }\end{array}$ & $\begin{array}{c}\text { No demando } \\
\text { de outonomia, } \\
\text { si clases }\end{array}$ & $\begin{array}{c}\text { Ni autonomia, } \\
\text { ni clases }\end{array}$ \\
\hline $\begin{array}{l}\text { Mucho } \\
\text { Bostante }\end{array}$ & $80,1 \%$ & $65,3 \%$ & $53,8 \%$ & $69,1 \%$ \\
\hline \begin{tabular}{l} 
Poco/Nada \\
\hline
\end{tabular} & $18,4 \%$ & $32,5 \%$ & $40,4 \%$ & $27,5 \%$ \\
\hline
\end{tabular}

El porcentaje de alumnos, dentro del conjunto de encuestas analizadas, que considera muy necesaria la presencia de libros y apuntes que le permitan estudiar autónomamente las distintas materias, asciende al 80,1\% entre quienes demandan autonomía tecnológica, es decir, entre quienes ha influido la posibilidad de autonomía y distancia de la enseñanza a distancia para matricularse en la UNED y dicen no precisar de clases presenciales para desarrollar sus estudios.

Queda finalmente abordar el grado de satisfacción con la institución de los alumnos que demandan tal autonomía tecnológica. A la vista de los resultados, cabría concluir que su demanda se encuentra bastante satisfecha. Entre los sectores diferenciados, es el de mayor porcentaje de quienes se encuentran mucho o bastante satisfechos como alumnos de la UNED, con un 90,8\%, frente a un 85,7\% de quienes demandan autonomía con clases presenciales o un 68,2\% entre quienes no demandan autonomía, pero sí clases presenciales, que puede considerarse el más distante de la UNED, al menos simbólicamente. De hecho, mientras el 42,2\% del último grupo de alumnos señaló que una de las razones que ha influido mucho o bastante para matricularse en la UNED ha sido el no haber podido acceder a otra Universidad; tal porcentaje desciende al 8,7\% entre los alumnos que aquí se han agrupado como demandantes de 
autonomía tecnológica. Como han observado Rickwood y Goddwin (2000), la demanda de autonomía en la enseñanza y, sobre todo, la enseñanza en autonomía, lleva aparejado un alto nivel de satisfacción con las instituciones que la ofrecen.

\section{CONCLUSIONES}

Los datos observados, obtenidos a partir de los respuestas a un cuestionario por una amplia muestra de alumnos, permiten algunas conclusiones de notable trascendencia, como es el aumento del peso de la autonomía entre los factores que llevan a los estudiantes a matricularse en la enseñanza superior a distancia ofertada por la UNED. Entre el conjunto de estudiantes matriculado en octubre de 1997, la demanda de autonomía es mayor entre los de más edad, los que tienen una ocupación laboral y quienes optan por disciplinas de Letras y Ciencias Sociales.

Al analizar la demanda de autonomía en función del curso en el que se matricula el alumno, parece apuntarse que la propia institución socializa en la independencia y autonomía en el estudio, pues se constata un importante peso de este factor como justificación de la opción por la UNED en el paso del cuarto al quinto curso de carrera universitaria. Tal demanda de autonomía, como toda autonomía, ha de considerarse relativa, ya que se sigue manteniendo un importante peso de la opinión a favor de la existencia de clases presenciales entre tales demandantes de autonomía en la enseñanza.

La demanda de autonomía tecnológica u opción por el seguimiento de una enseñanza que prescinda de las clases presenciales, se concentra en sectores urbanos y entre quienes ocupan las mejores posiciones en la estructura social, dentro del conjunto del total de alumnos matriculados. Ha de subrayarse que el alumno que demanda autonomía tecnológica es el simbólicamente más cercano a la UNED, como institución de enseñanza superior a distancia.

Ante la ausencia de datos más refinados sobre el uso de medios de comunicación para la enseñanza por parte de los alumnos, cabe concluir que la demanda tecnológica se fundamenta especialmente en material escrito. En el futuro, se puede esperar que la demanda de autonomía tecnológica deje de concentrarse en el material escrito tradicional, pasando a apoyarse en otros medios.

También cabe vaticinar un crecimiento de la demanda de autonomía en general, especialmente vinculada con la evolución de las denominadas sociedades desarrolladas. En la versión de la autonomía aquí abordada, la tecnológica, el proceso de autonomización tendrá consecuencias sobre el conjunto del sistema educativo y los modelos de aprendizaje. Al respecto, el informe Dearing sobre la enseñanza superior en Gran Bretaña, incluyendo la enseñanza presencial, apunta los importantes cambios que se producirán en el sistema educativo con el crecimiento del énfasis en el aprendizaje independiente (punto 8.12 del informe).

En otro nivel, las instituciones de educación a distancia deben preguntarse sobre su grado de preparación para gestionar la autonomía en la enseñanza. Le- bel (1995) resalta en un estudio, cuya base muestral (14 tutores) se reconoce inviable para la generalización, la relativa incapacidad de los tutores de una institución de enseñanza a distancia de Quebec para enfrentarse a la autonomía de sus alumnos. Ha de tenerse en cuenta, como se señaló en el primer apartado de este escrito, que la autonomía no es sólo un producto de la tecnología. Exige, también, un contexto social e institucional para su desarrollo, lo que incluye tecnología, tanto relativa a los procedimientos docentes, como a la disponibilidad de los alumnos de los soportes técnicos y cognitivos necesarios.

A su vez, el crecimiento de las posibilidades de autonomía tecnológica, derivado de la extensión de los medios digitales, obliga a cambios didácticos radicales (Peters 1998) en la propia enseñanza a distancia. Se abre, siguiendo al autor alemán, una nueva generación en la enseñanza a distancia. Una generación que está todavía por hacerse. Si la autonomía no encuentra un nuevo modelo didáctico, nos encontraremos con que la mejor garantía de aquella es algo tan relativamente tradicional y, a la vez, moderno, como son los exámenes prescritos, que permiten que el alumno se prepare autónomamente para las posibles preguntas. Nos encontraremos con una autonomía enclaustrada en los límites del examen.

\section{REFERENCIAS BIBLIOGRÁFICAS}

BATESON, G. (1990): Steps toan ecology of Mmd, Toronto: Ballantine (e.o. 1972). 
BOURDIEU, P. (1988): La distinción, Madrid: Taurus.

BECK, U. (1997): “La reinvención de la política: Hacia una teoría de la modernización reflexiva”, en U. Beck, A. Giddens y S. Lasch, Modernización reflexiva. Política, tradición y estética en el orden social moderno, Madrid: Alianza.

DANIEL, J. y C. MARQUIS (1979): “Interaction and independence: getting the mixture right”, Teaching at a Distance, 14, pp. 2 9-44.

DESCARTES, R. (1983): Discurso del método, Madrid: Aguilar (e.o. 1637).

DUBY, G. (1993): “La vida privada en las familias aristocráticas de la Francia feudal. Convivialidad”, en P. Ariés y G. Duby (dir), Historia de la vida privada. De la Europa feudal al Renacimiento, Barcelona: Círculo de Lectores.

ELIAS, N. (1987): El proceso de civilización: investigaciones sociogenéticas y psicogenéticas, México: Fondo de Cultura Económica.

EVANS, T. y D. NATION (1999): “De la necesidad de la teoría en la enseñanza abierta y a distancia”, en E. Martín Rodríguez y M. Ahijado Quintillán (coords.): La educación a distancia en tiempos de cambios: nuevas generaciones, viejos conflictos, Madrid: Ed. de la Torre, pp. 61-84.

GIDDENS, A. (1991): The constitution of society, Cambridge: Polity Press.

GIDDENS, A. (1995): Modernidad e identidad del yo, Barcelona: Península.

GIDDENS, A. (1997): “Riesgo, confianza, reflexividad”, en U. Beck, A. Giddens y S. Lasch, Modernización reflexiva. Política, tradición y estética en el orden social moderno, Madrid: Alianza.

HAVELOCK, E. A. (1963): Preface to Plato, Cambridge (Mass.): Belknap Press of Harvard Universitv Press.

HAVELOCK, E. A. (1982): The literate revolution in Greece and its cultural consequences, PrincePrinceton Universtiy Press.

HOLMIBERG, B. (1987): "Student autonomy in theory and practice”, Journal of Innovative Higher Pducation, 4 (1-2), PP. 4-8.

ICE-UNED (1982): Los alumnos de la UNED, Madrid: UNED

INNIS, H. (1971a): Empire and Communication, Toronto: University of Toronto Press (e.o. 1950).

INNIS, H. (1971b): The bias of communication, Toronto: University of Toronto Press (e.o. 1951).

IUED (UNED) (1998): Anuario estadístico de la UNED. Curso 1994-1995, Madrid: UNED.

KERCKHOVE, D. (1999): La piel de la cultura, Barcelona: Gedisa.

LEBEL, C. (1995): “Le tuteur et l'autonomie de l'étudiant á distance”, Journal of Distance Education, vol. 10 (1), primavera, pp. 5-24.

LUHMANN, N. (1996): La Ciencia de la Sociedad, México: Anthropos-Iberoamericana.

McLUHAN, M. (1972): La galaxia Gutenberg. Génesis del “horno typographycus”, Madrid: Aguilar (e.o. 1962).

McLUHAN, M. (1973): La comprensión de los medios como extensión del hombre, México: Diana (e.o. 1964). 
M00RE, M. (1983): “Towards a theory of independent learning and teaching”, Journal of Higher Education, 12, pp. 661-679.

MORIN, E. (1983): El método II. La vida de la vida, Madrid: Cátedra.

Morley, D. (1998): “El posmodernismo: una guía básica”, en J. Curran, D. Morley, y. Walkerdine (comps), Estudios culturales y comunicación, Barcelona: Paidós.

ONG, W. (1982): Orality and Literacy: the technologizing of the world, Londres: Methuen.

OUTRAM, D. (1989): The Body and the French Revolution: Sex, Class and Political Culture, New Haven: Yale University Press.

PAUL, R. (1999): "Hacia una autonomía del alumno: nueva pauta para medir los logros de una institución de enseñanza abierta”, en E. Martín Rodríguez y M. Ahijado Quintillán (coords.): La educación a distancia en tiempos de cambios: nuevas generaciones, viejos conflictos, Madrid: Ed.

de la Torre, pp. 43-60.

PEYREFITTE, A. (1997): Milagros económicos, Barcelona: Andrés Bello.

PÉREZ JUSTE, R., L.GARCÍA, S. GENTO, J.A. GIL Y J. M. LUZÓN (1999): ¿Qué piensan los alumnos de la UNED de su propia Universidad?, Madrid: UNED.

PETERS, 0. (1983): "Distance teaching and industrial production: a comparative interpretation in outline”, en D. Sewart, D. Keegan y B Holmberg (eds.): Distance Education: international perspectives, Londres: Croom Helm, pp. 95-122.

PETERS, 0. (1998): “Die Autonomie der Lernenden und ihre Bedeutung für das Fernstudium”, IAD, año $\mathrm{X}, \mathrm{n}^{\circ} .10 / 1$ 1, PP. 57-82.

RICKWOOD, P. y y. GOODWIN (2000): “Travellers' Tales: reflections on the way to learner autonomy”, Open Learning, vol.15, $n^{\circ} .1$, pp. 47-55.

ROWNTREE, D. (1990): Teaching Through Self-Instruction: How to Develop Open Learning Materials, Londres: Kogan Page.

SEWART, D., D. Keegan y B. Holmberg (eds.) (1983): Distance Education: international perspectives, Londres: Croom Helm.

TURNER, B. S. (1996): The Body \& Society, Londres: Sage.

UNED (1981): Lcs primeros licenciado.s de la IJWFfl, MaHrid TJMF,D

VARELA, F. (1979). Principle.s of biulogival uutunwny, Madrid: Catedia.

WEBER, M. (1979): Economía y Sociedad, México: Fondo de Cultura Económica.

ZINNECKER, 3. (1972): "Die Neukonstituierung der Erzvetungswissenschaft als Sozialtechnologie”, Beitrüge ur Bildungstechnologie, 1, págs. 77-89.

\section{PERFIL ACADÉMICO-PROFESIONAL}

Javier Callejo Gallego es actualmente Director Adjunto del Instituto Universitario de Educación a Distancia (UNED). Es doctor en Sociología y licenciado en CC. de la Información (Periodismo). Profesor titular de Técnicas de Investigación Social en la Facultad de CC. Políticas y Sociología de la UNED. Su línea de investigación se ha centrado en medios de comunicación, sociología del consumo y sociología de la educación. Autor, entre otros, de La audiencia activa (Madrid: Centro de Investigaciones Sociológicas, 1995). 


\section{Javier Cadejo}

Departamento de Sociología 1. Teoría, Métodos y Cambio Social.

Facultad de Ciencias Políticas y Sociología

UNED

c/Obispo Trejo s/n

28040 Madrid (España)

Telf: + 34913987065

Dirección electrónica: mcallejo@poli.uned.es 